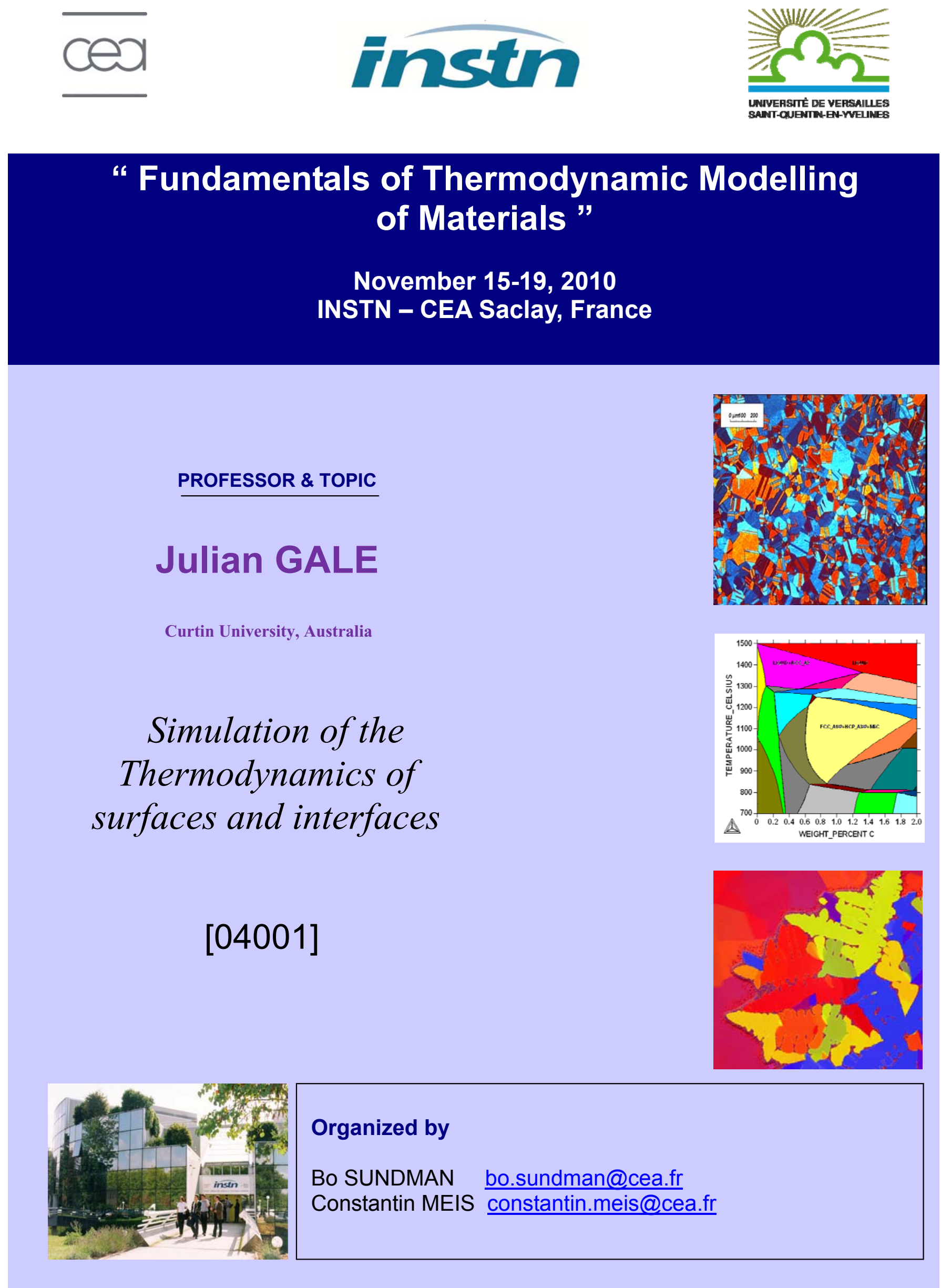

This is an Open Access article distributed under the terms of the Creative Commons Attribution-Noncommercial License 3.0, which permits unrestricted use, distribution, and reproduction in any noncommercial medium, provided the original work is properly cited. 


\section{Simulation of the Thermodynamics of Surfaces and Interfaces}
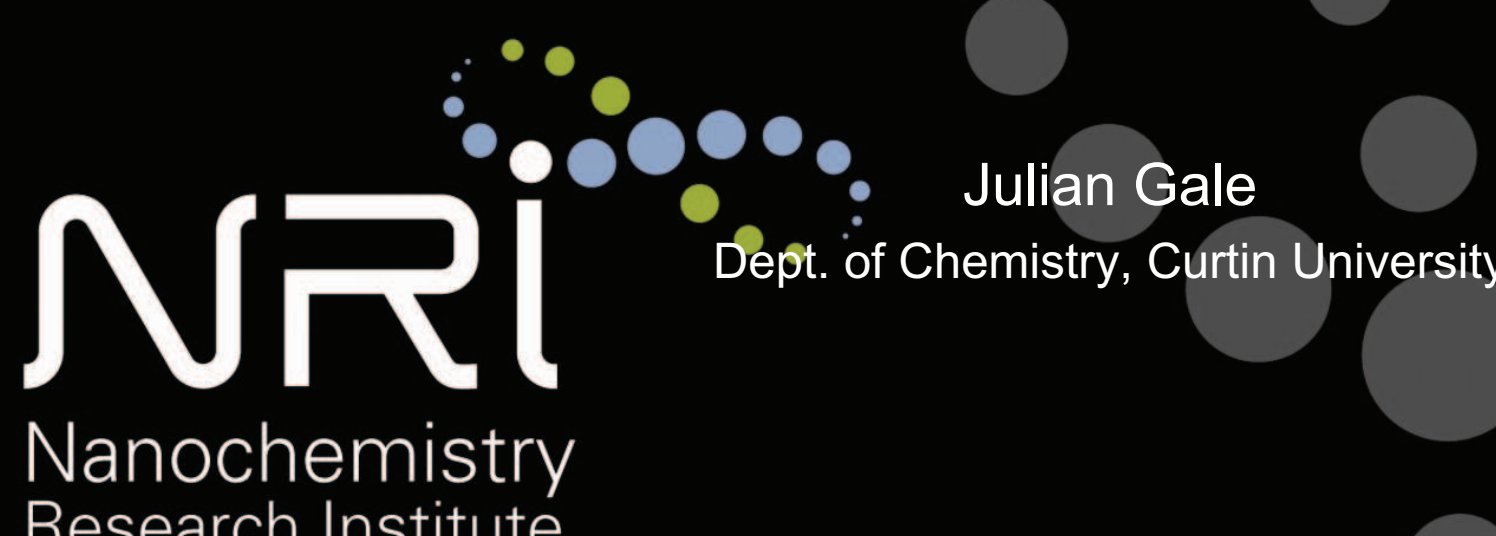

Dept. of Chemistry, Curtin University

Research Institute
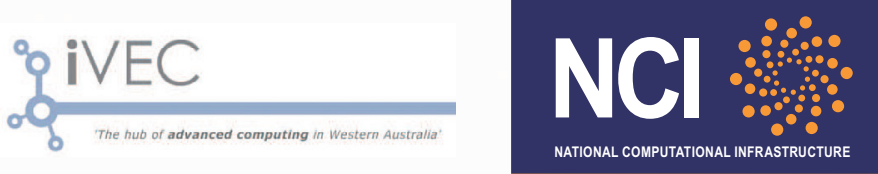

Curtin晴

Australian Research Council

\section{Thermodynamics of the Solution} Interface: Nucleation, Nanoparticles, Polymorphism and Morphology

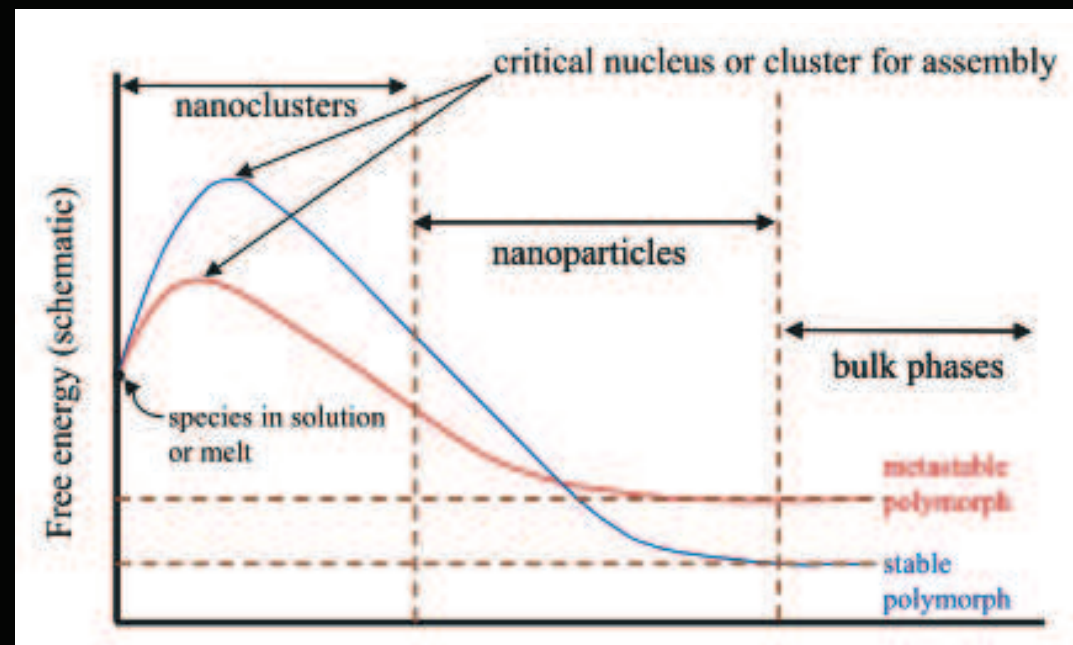

Particle radius

Alex Navrotsky, PNAS, 101, 12096 (2004) 


\section{Surface Simulation}

- Surface in vacuo

- Lattice dynamics is generally best

- Solid-liquid interface

- Molecular dynamics is appropriate

- Solid-solid interface

- Can be most challenging case

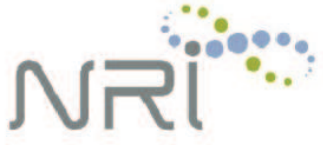

\section{Equilibrium Morphology}

- Compute the surface energy

$$
\Delta U_{S E}=\frac{\left(U_{\text {surface }}-U_{\text {bulk }}\right)}{A}
$$

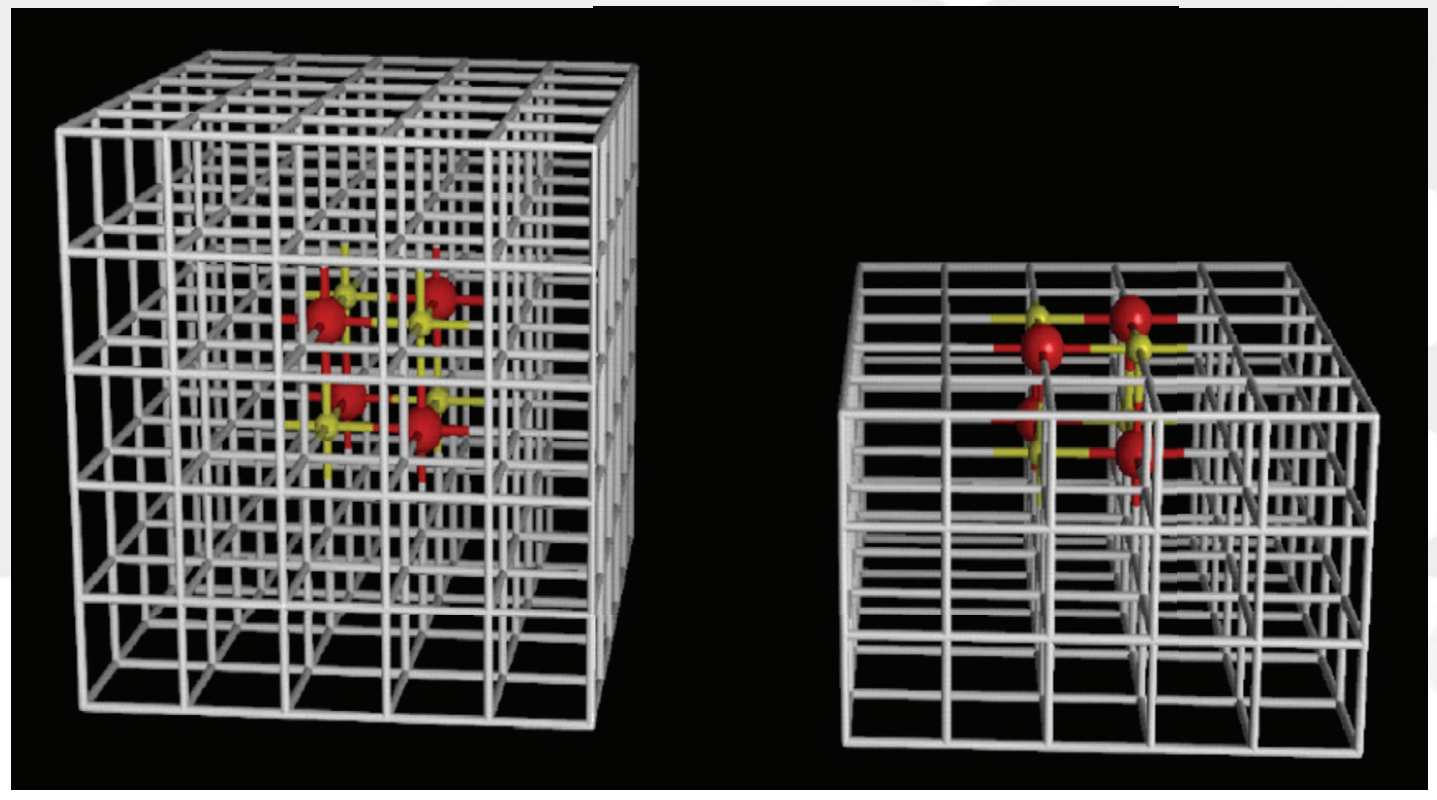




\section{Growth Morphology}

- Quasi-kinetic

- Uses the attachment energy

- $U_{\text {att }}$ defined as the energy per molecule released when a new slice of depth $d_{\mathrm{hkl}}$ is attached to the crystal face.

- Small attachment energy yields large faces

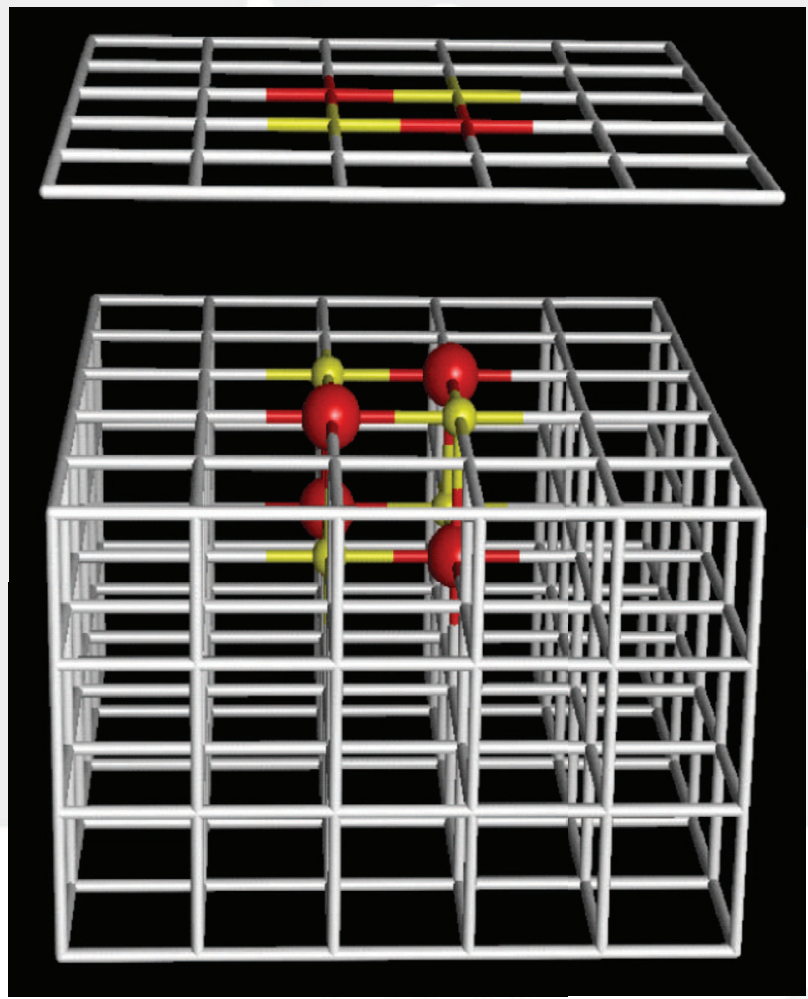

\section{Surface Simulation Model}

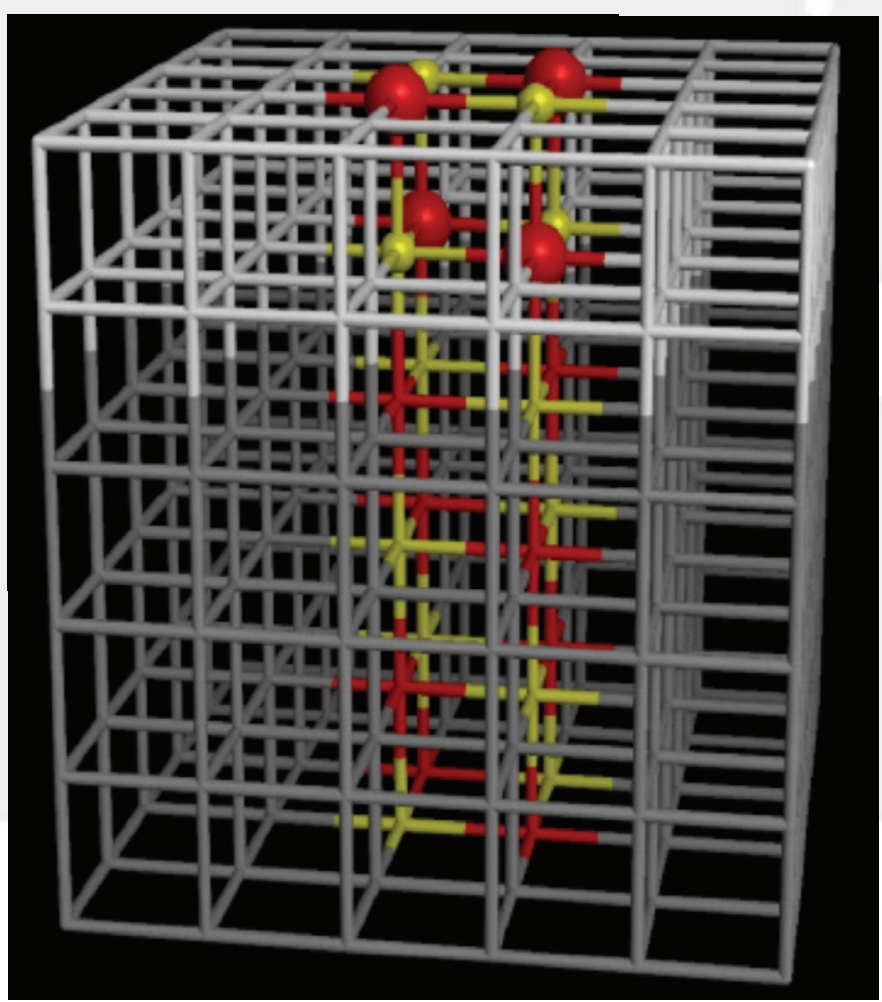

Two region

strategy

OR

Slab

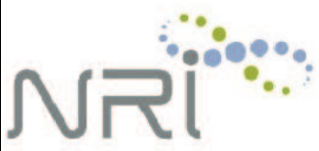




\section{Surface Types}

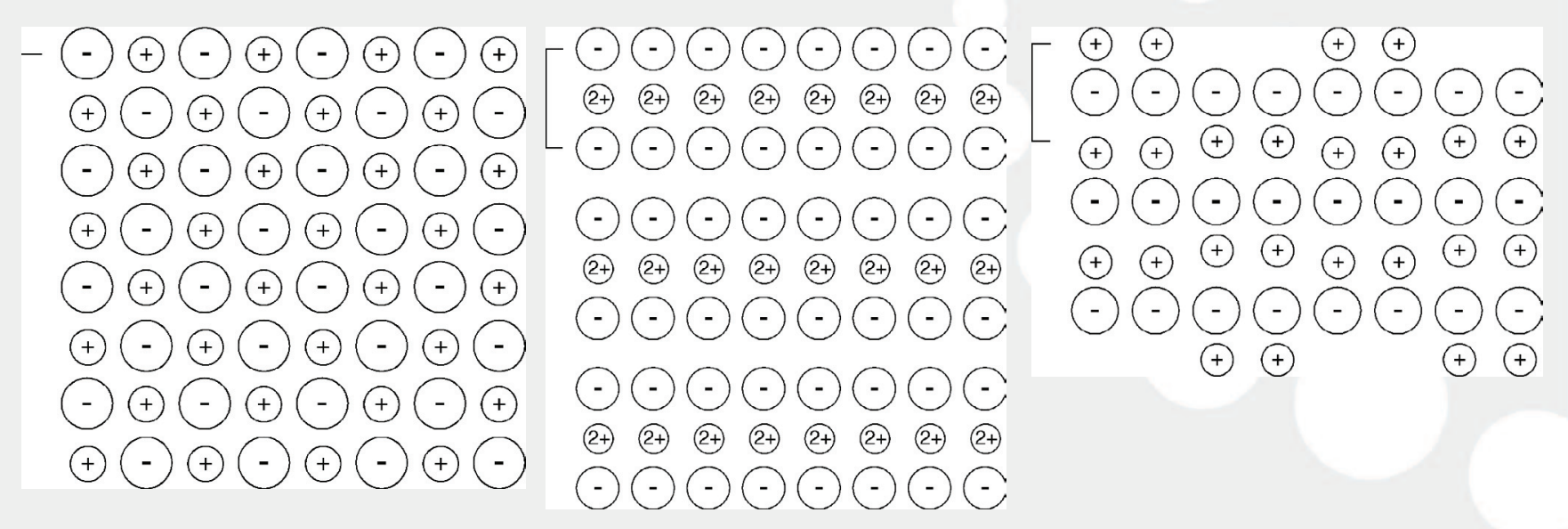

Type 1

Type 2a

Type 2b

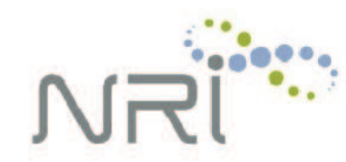

\section{Morphology of Gibbsite: $\mathrm{Al}(\mathrm{OH})_{3}$}
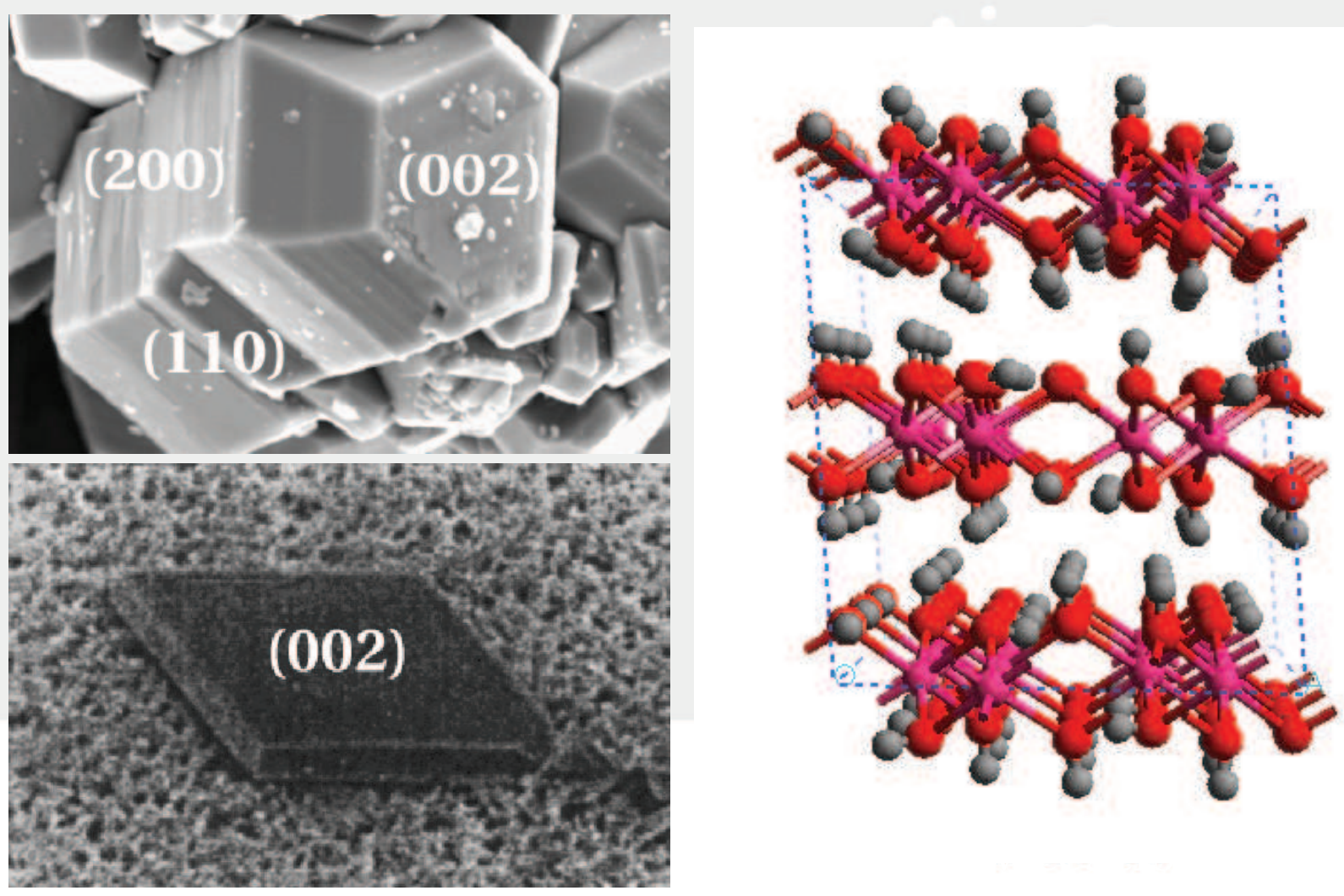


\section{Calculated Gibbsite Morphology}
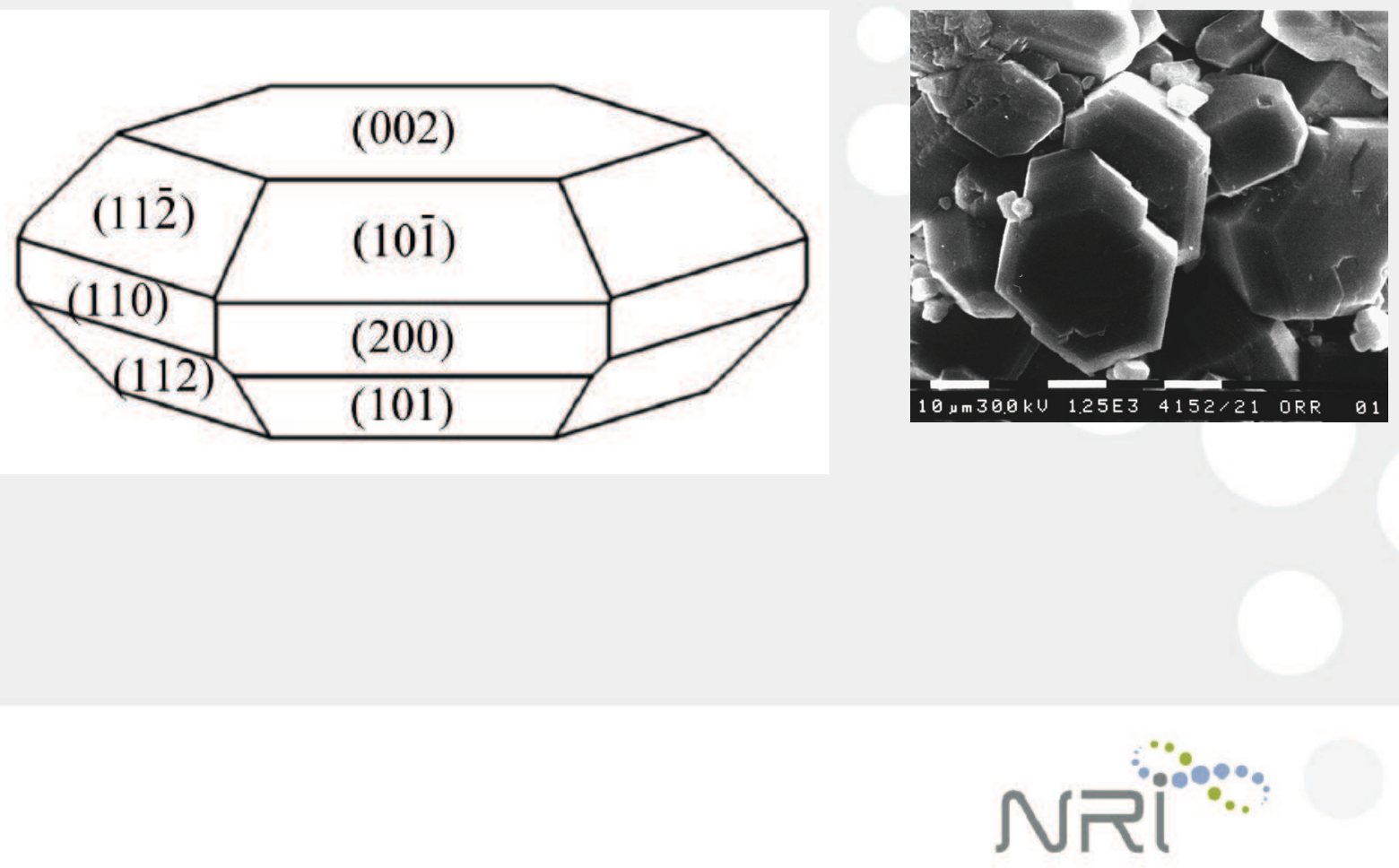

\section{Sodium Incorporation}

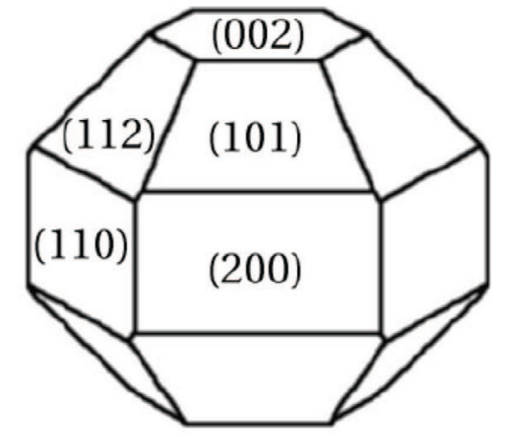

Low levels of incorporation

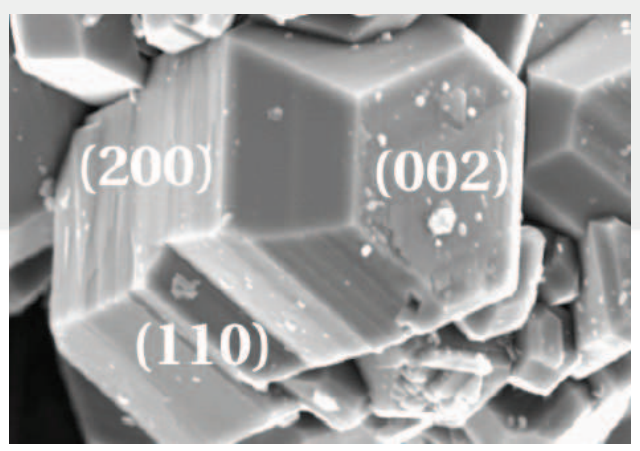

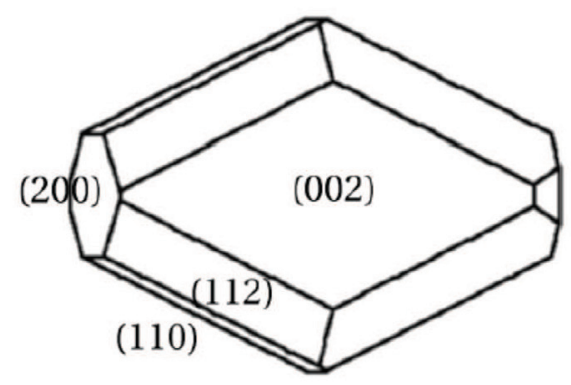

High levels of incorporation $\left(\mathrm{Na}^{+}\right.$only)

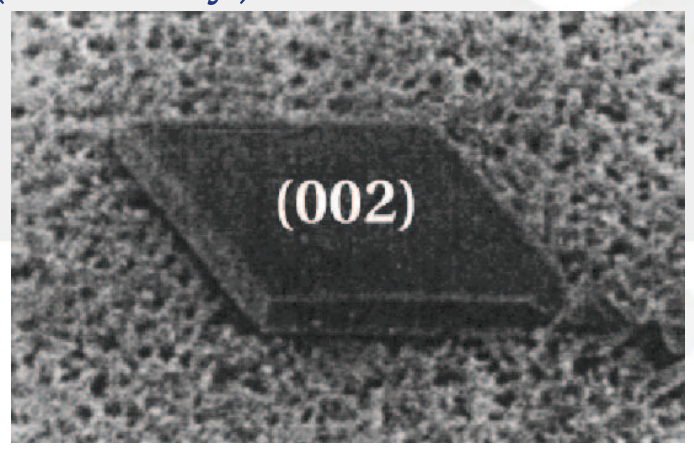




\section{(10-14) Surface of Calcite}
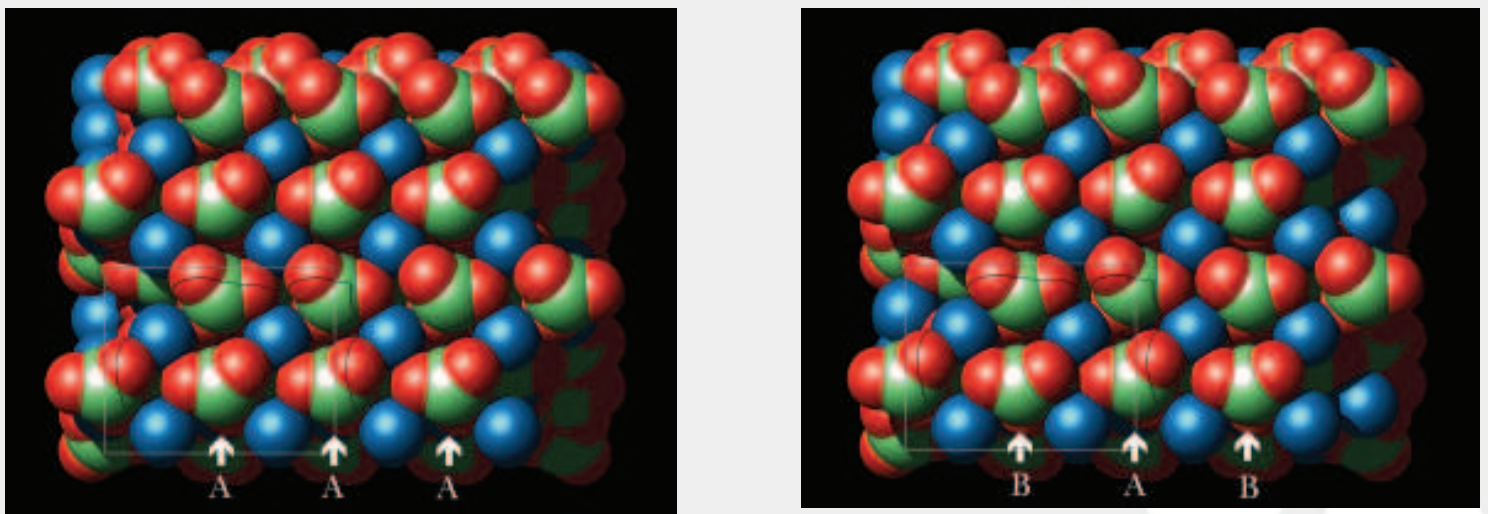

Imaginary mode at $(1 / 2,0)$ in $\mathrm{BZ}$

Rohl et al, Am. Miner., 88, 921 (2003)
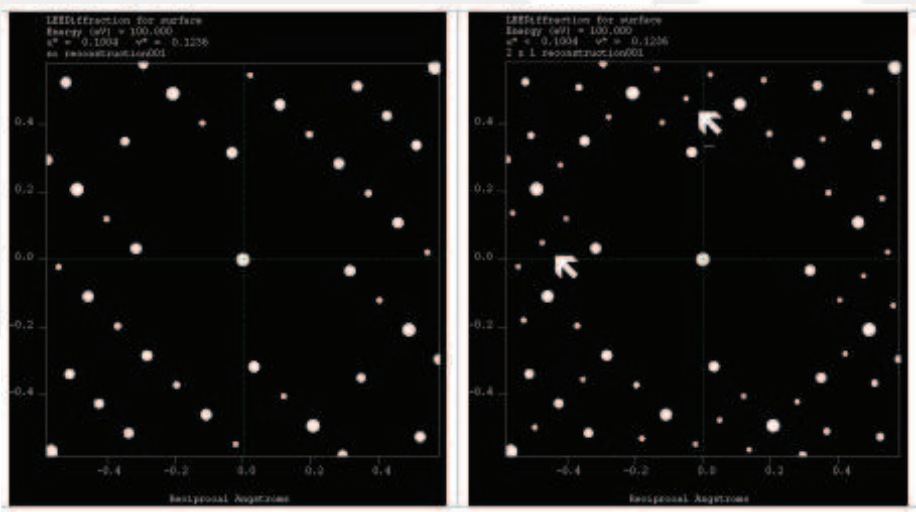

\section{Surface Thermodynamics}

- Morphology only probes relative surface energy

- Calcite basal plane:

- Experimental:

- $0.347 \pm 0.045 \mathrm{~J} / \mathrm{m}^{2}$ (Int. J. Rock Mech., 5, 253 (1968))

- $1.4 \pm 0.4 \mathrm{~J} / \mathrm{m}^{2}$ (A. Navrotsky, Abstract GSA Meeting (2010))

- $\Delta \mathrm{H}$ immersion $=-0.535 \mathrm{~J} / \mathrm{m}^{2}$ (Coll. Surf. A, 80, 261 (1993))

- Calculated:

- $0.711 \mathrm{~J} / \mathrm{m}^{2}$ (Raiteri \& Gale, 2010)

- $0.261 \mathrm{~J} / \mathrm{m}^{2}$ (COSMIC Solvation, Gale \& Rohl, 2007)

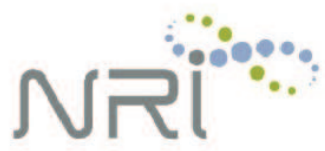




\section{Grain Boundaries}

Four region strategy: 2-1-1'-2'

General interfaces possible for mismatch $<5 \%$

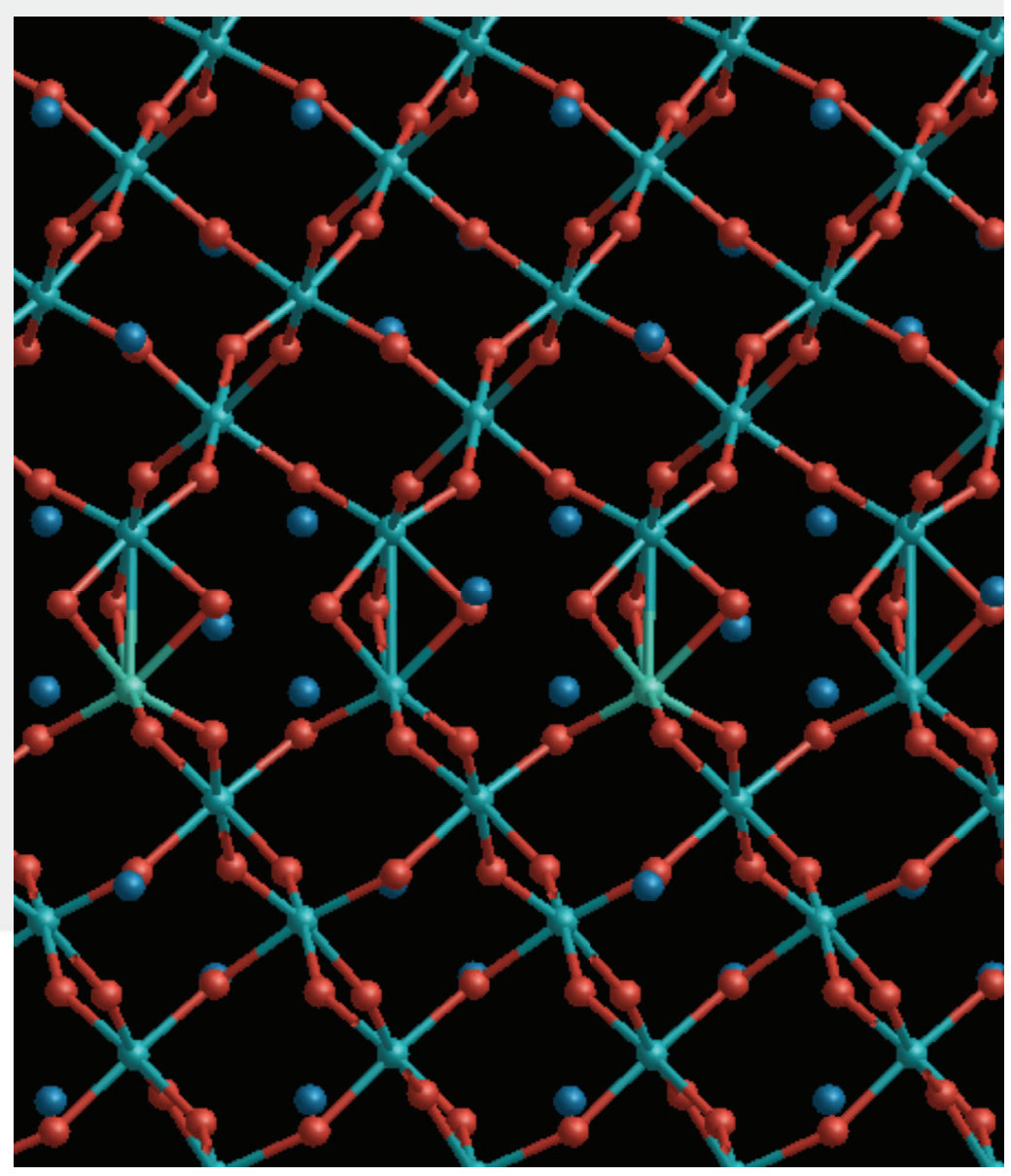

Barite $\left(\mathrm{BaSO}_{4}\right)$

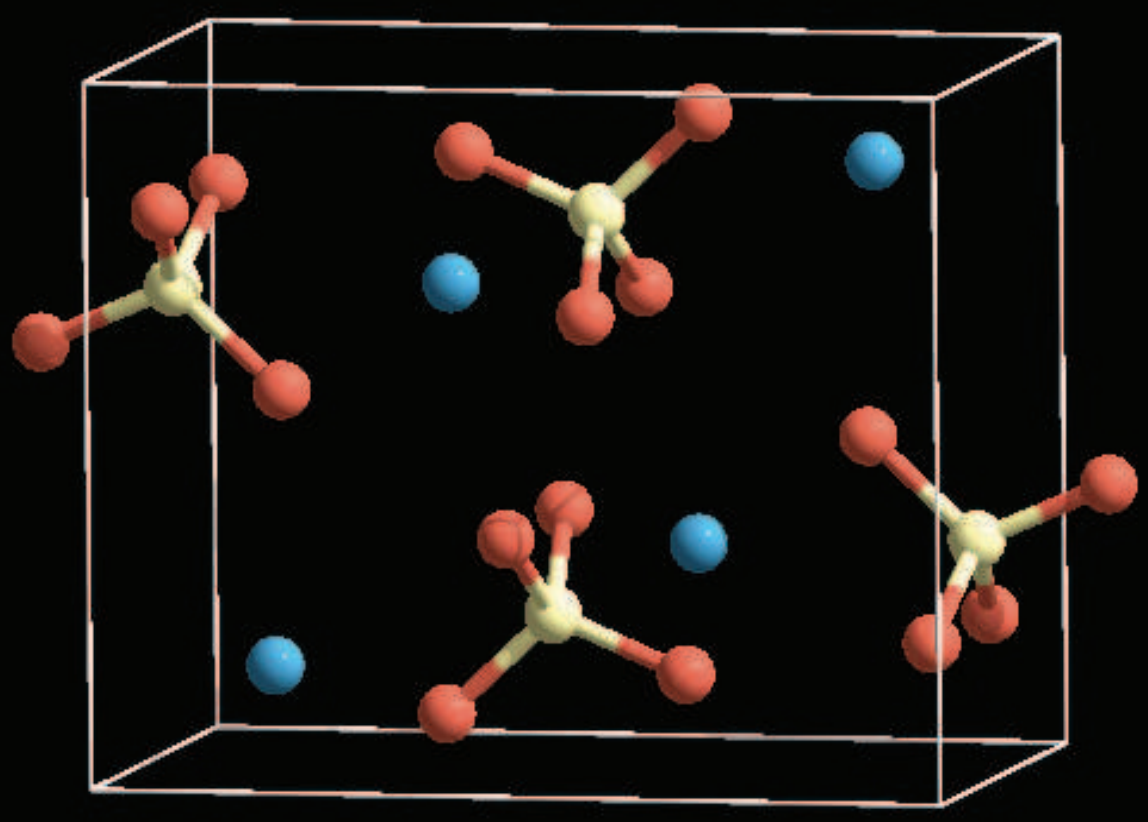


(001)

\section{Surfaces of barite}
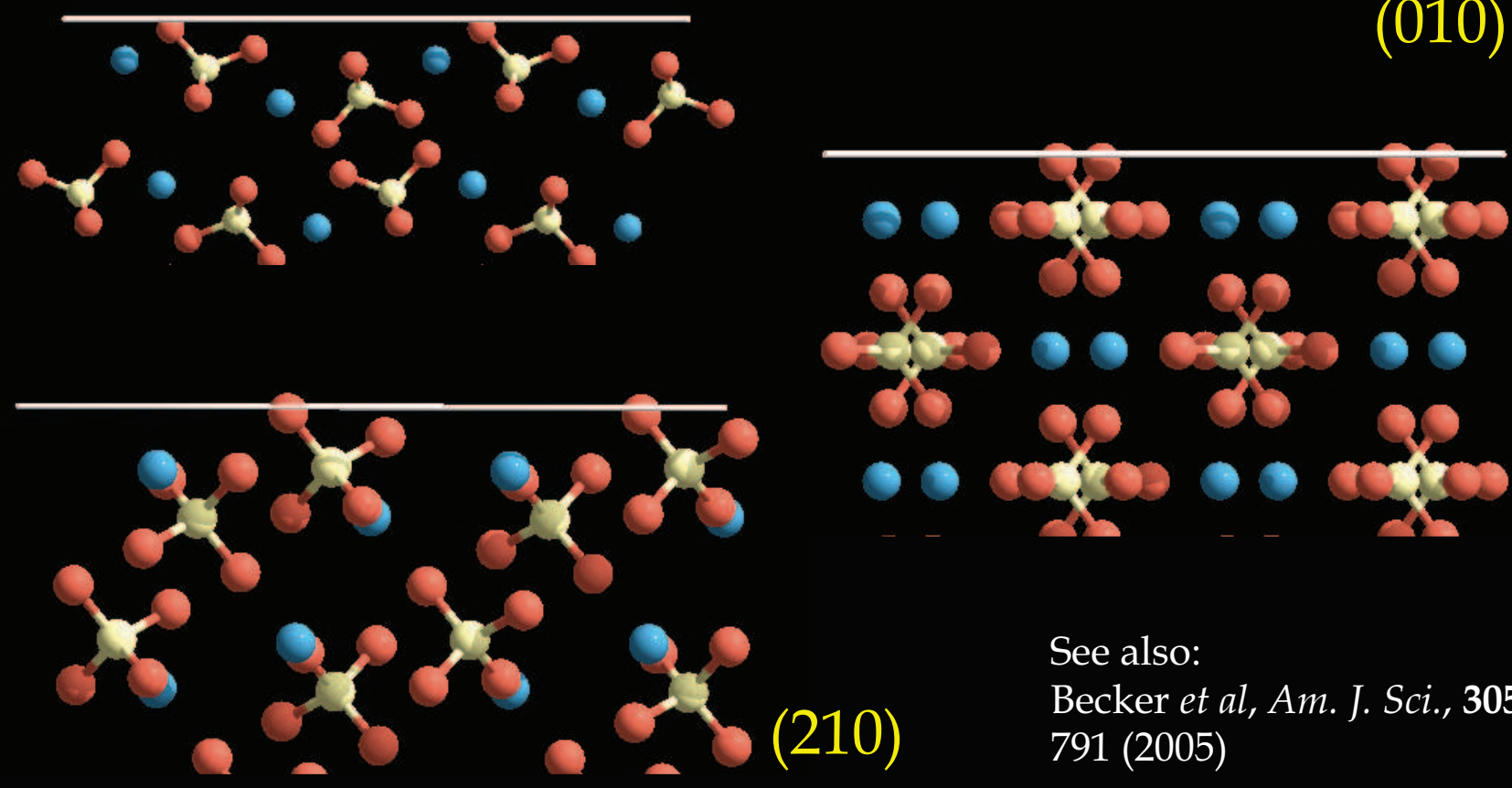

See also:

Becker et al, Am. J. Sci., 305, 791 (2005)

\section{The morphology of barite}

$(-2-10)$

$(0-20)$

(2-10)

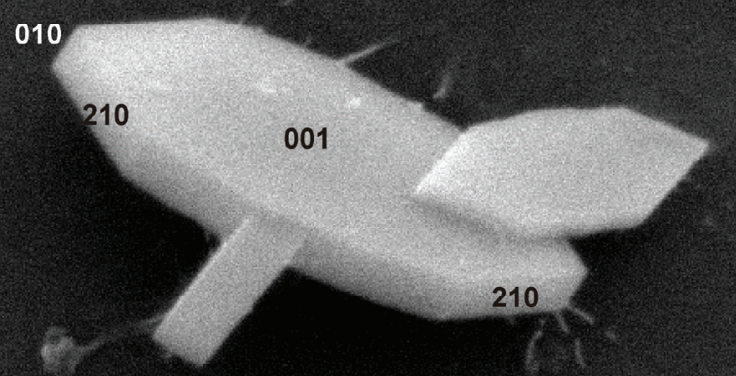

Solvated surface energies:

(001) 241(18) $\mathrm{mJm}^{-2}$

(010) 193(15) $\mathrm{mJm}^{-2}$

(210) 194(15) $\mathrm{mJm}^{-2}$ 
A 100 ns in the life of a solvated (010) surface of barite
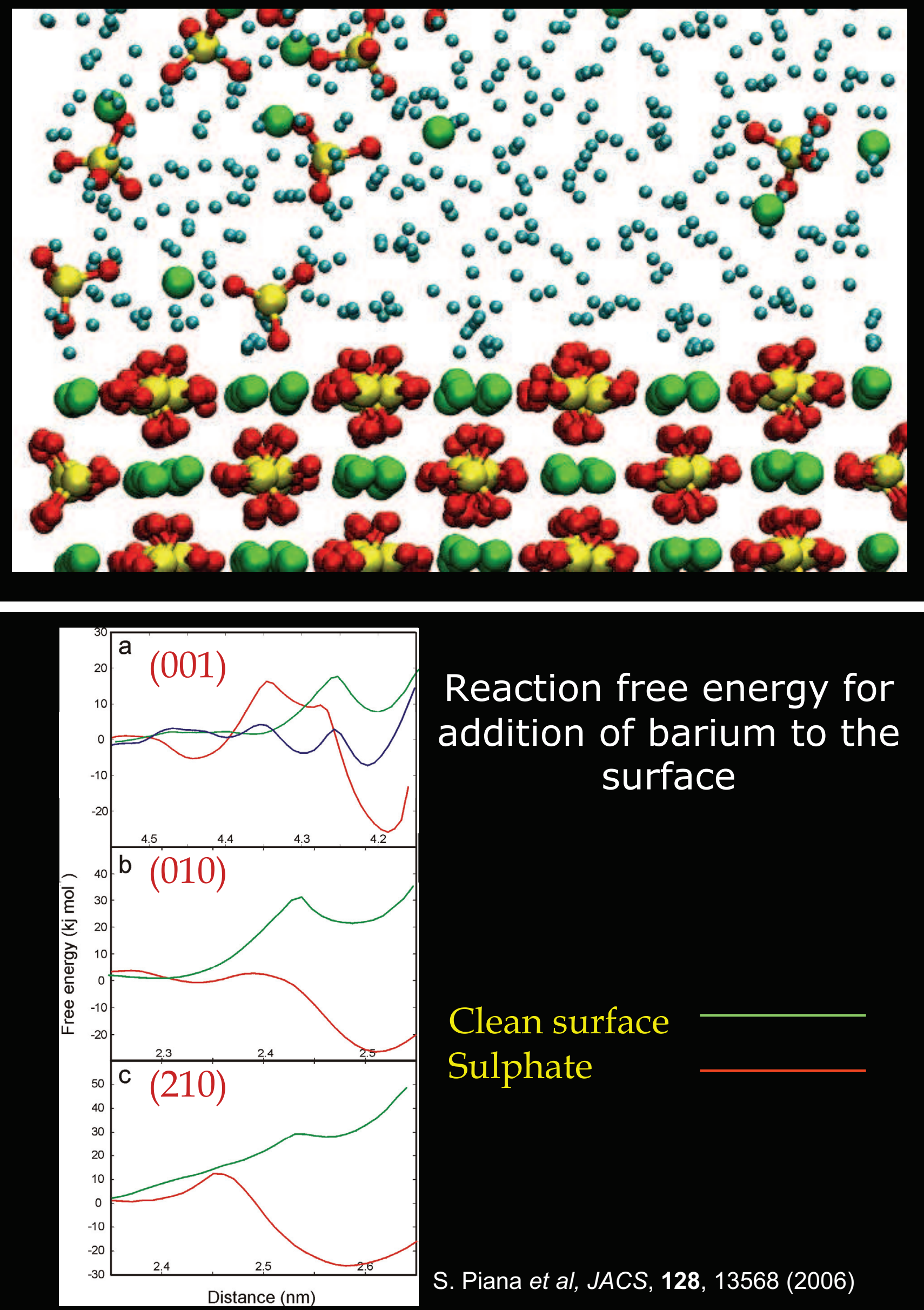

Reaction free energy for addition of barium to the surface

Clean surface

Sulphate

S. Piana et al, JACS, 128, 13568 (2006) 


\section{Influence of aspartic acid on barite}

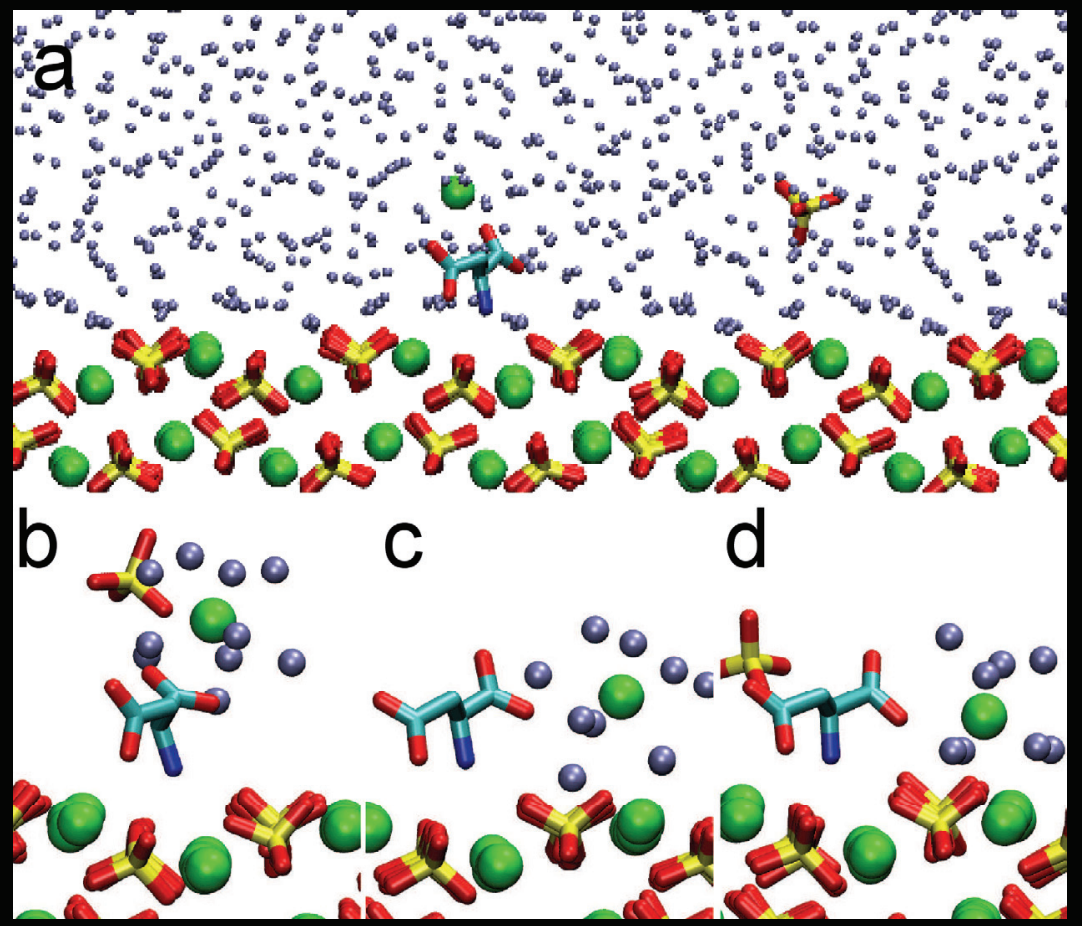

S. Piana, F. Jones, JDG, CrystEngComm, 9, 1187 (2007)

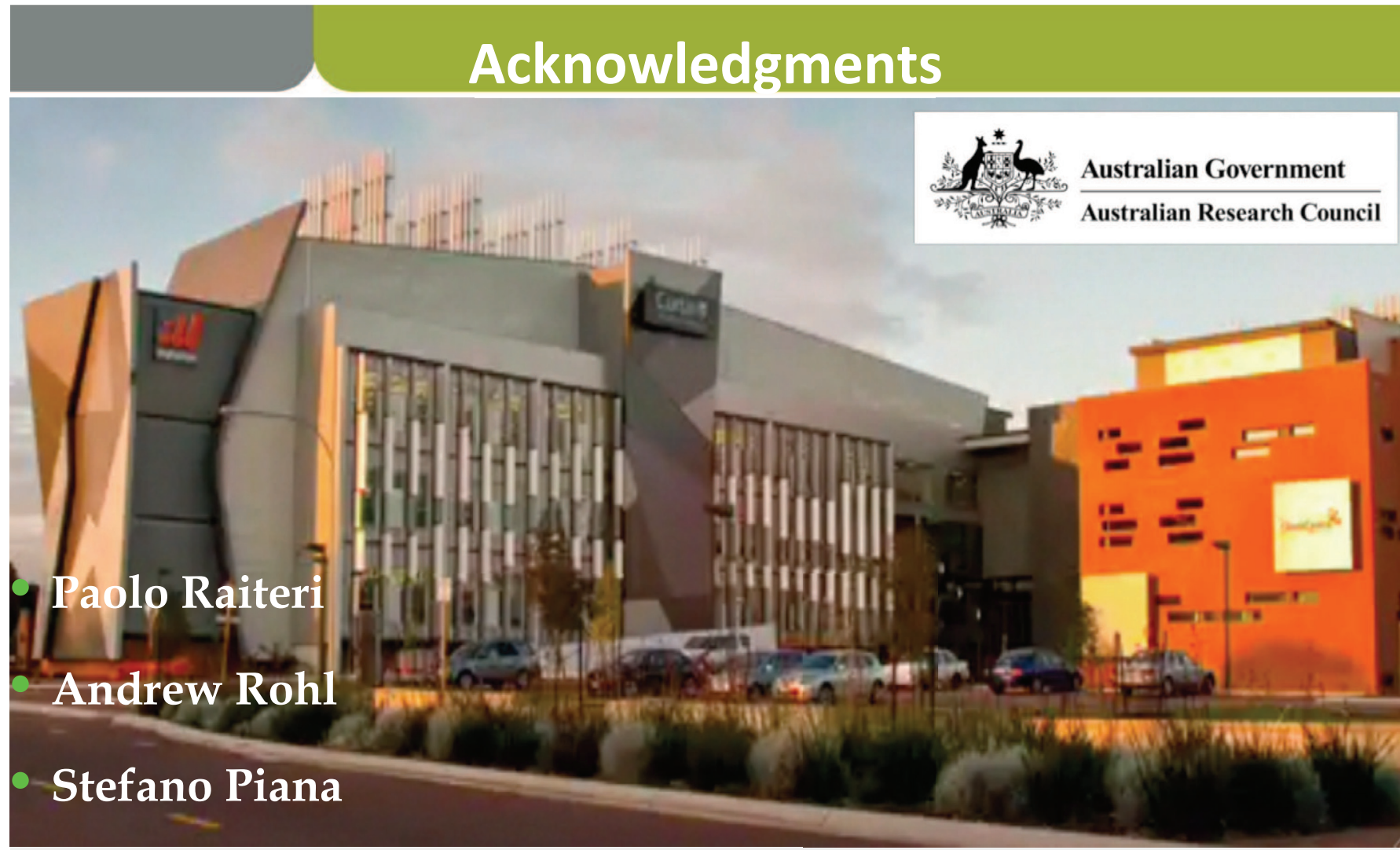

iVEC

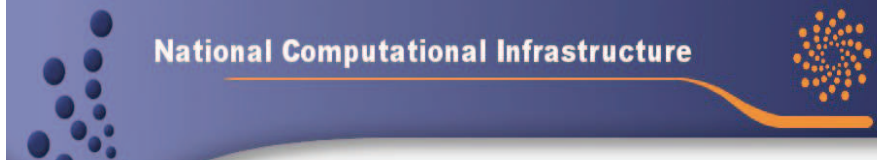

